\title{
Sequences associated to elliptic curves with non-cyclic torsion subgroup
}

\author{
Betül Gezer (D) \\ Bursa Uludag University, Faculty of Science, Department of Mathematics, Görükle, 16059, Bursa, Turkey
}

\begin{abstract}
Let $E$ be an elliptic curve defined over $K$ given by a Weierstrass equation and let $P=$ $(x, y) \in E(K)$ be a point. Then for each $n \geq 1$ we can write the $x$ - and $y$-coordinates of the point $[n] P$ as

$$
[n] P=\left(\frac{G_{n}(P)}{F_{n}^{2}(P)}, \frac{H_{n}(P)}{F_{n}^{3}(P)}\right)
$$

where $F_{n}, G_{n}$, and $H_{n} \in K[x, y]$ are division polynomials of $E$. In this work we give explicit formulas for sequences

$$
\left(F_{n}(P)\right)_{n \geq 0},\left(G_{n}(P)\right)_{n \geq 0}, \text { and }\left(H_{n}(P)\right)_{n \geq 0}
$$

associated to an elliptic curve $E$ defined over $\mathbb{Q}$ with non-cyclic torsion subgroup. As applications we give similar formulas for elliptic divisibility sequences associated to elliptic curves with non-cyclic torsion subgroup and determine square terms in these sequences.
\end{abstract}

Mathematics Subject Classification (2010). 14H52, 11B37, 11G05

Keywords. elliptic curves, division polynomials, elliptic divisibility sequences, squares

\section{Introduction}

Let $E$ be an elliptic curve defined over a field $K$ given by a Weierstrass equation

$$
E: y^{2}+a_{1} x y+a_{3} y=x^{3}+a_{2} x^{2}+a_{4} x+a_{6} .
$$

Let $E(K)$ be the group of $K$-rational points on $E$, and let $\mathcal{O}$ denote the point at infinity, the identity for the group $E(K)$. For background on elliptic curves we refer the reader [24] and [26]. The $n$-division polynomial $F_{n} \in K[x, y]$ for the elliptic curve $E$ evaluated at a point $P=(x, y) \in E(K)$ is defined using the initial values

$$
\begin{aligned}
F_{0}(P) & =0 \\
F_{1}(P) & =1 \\
F_{2}(P) & =2 y+a_{1} x+a_{3}, \\
F_{3}(P) & =3 x^{4}+b_{2} x^{3}+3 b_{4} x^{2}+3 b_{6} x+b_{8}, \\
F_{4}(P) & =F_{2}(P)\left(2 x^{6}+b_{2} x^{5}+5 b_{4} x^{4}+10 b_{6} x^{3}+10 b_{8} x^{2}\right. \\
& \left.+\left(b_{2} b_{8}-b_{4} b_{6}\right) x+\left(b_{4} b_{8}-b_{6}^{2}\right)\right),
\end{aligned}
$$

Email address: betulgezer@uludag.edu.tr

Received: 26.09.2018; Accepted: 09.11.2019 
where

and

$$
b_{2}=a_{1}^{2}+4 a_{2}, \quad b_{4}=2 a_{4}+a_{1} a_{3}, \quad b_{6}=a_{3}^{2}+4 a_{6},
$$

and by the formulas

$$
b_{8}=a_{1}^{2} a_{6}+4 a_{2} a_{6}-a_{1} a_{3} a_{4}+a_{2} a_{3}^{2}-a_{4}^{2},
$$

$$
\begin{aligned}
F_{2 n+1}(P)= & F_{n+2}(P) F_{n}(P)^{3}-F_{n-1}(P) F_{n+1}(P)^{3}, \quad \text { for } n \geq 2, \\
F_{2 n}(P)= & \left(F_{n-1}(P)^{2} F(P) F_{n+2}(P)\right. \\
& \left.-F_{n-2}(P) F_{n}(P) F_{n+1}(P)^{3}\right)\left(F_{2}(P)\right)^{-1}, \quad \text { for } n \geq 3 .
\end{aligned}
$$

The $n$-division polynomial $F_{n} \in K[x, y]$ has divisor

$$
\operatorname{div}\left(F_{n}\right)=\sum_{T \in E[n]}(T)-n^{2}(\mathcal{O})
$$

where $E[n]$ is the $n$-torsion subgroup of $E(K)$, so it vanishes exactly at $n$-torsion points and has a pole at $\mathcal{O}$. These polynomials arise in expressing the coordinates of $[n] P$ in terms of a point $P \in E(K)$ (with $\operatorname{char}(K) \neq 2$ ), that is the point $[n] P$ can be given by

$$
[n] P=\left(\frac{G_{n}(P)}{F_{n}(P)^{2}}, \frac{H_{n}(P)}{F_{n}(P)^{3}}\right)
$$

for division polynomials $F_{n}, G_{n}$, and $H_{n} \in K[x, y]$, where $G_{n}(P)$ and $F_{n}(P)^{2}$ are relatively prime. Furthermore, the polynomials $G_{n}(P)$ and $H_{n}(P)$ are given by the recurrence relations

$$
\begin{aligned}
& G_{0}(P)=1, G_{1}(P)=x \\
& H_{0}(P)=1, H_{1}(P)=y
\end{aligned}
$$

and

$$
\begin{aligned}
G_{n}(P)= & x F_{n}(P)^{2}-F_{n+1}(P) F_{n-1}(P), \\
H_{n}(P)= & \left(F_{n-1}(P)^{2} F_{n+2}(P)-F_{n-2}(P) F_{n+1}(P)^{2}\right. \\
& \left.-F_{2}(P) F_{n}(P)\left(a_{1} G_{n}(P)+a_{3} F_{n}(P)\right)\right)\left(2 F_{2}(P)\right)^{-1},
\end{aligned}
$$

for all $n \geq 2$. The division polynomials $F_{n}$ satisfy the more general recurrence relation

$$
F_{m+n}(P) F_{m-n}(P)=F_{m+1}(P) F_{m-1}(P) F_{n}(P)^{2}-F_{n+1}(P) F_{n-1}(P) F_{m}(P)^{2}
$$

for all $m \geq n \geq 1$. We also note that the recurrences (1.2) can be obtained from this formula.

Division polynomials appear in the theory of elliptic functions, the theory of elliptic curves, and the theory of elliptic divisibility sequences. Ward studied arithmetic properties of these polynomials in a series of papers [30,31]. Ayad [1] studied periodicity properties of the sequence $\left(F_{n}(P)\right)_{n \geq 0}$ of values of the division polynomials of an elliptic curve $E$ evaluated at a point $P$. Silverman [23] used a lift to characteristic zero and the Lefschetz principle to prove that the sequence $\left(F_{n}(P)\right)_{n \geq 0}$ of values of division polynomials is purely periodic, which is a generalization a result of Ward for elliptic divisibility sequences. Silverman [23] also considered $p$-adic properties of the sequence $\left(F_{n}(P)\right)_{n \geq 0}$, and proved the existence and algebraicity of the $p$-adic limit of certain subsequences of the sequence $\left(F_{n}(P)\right)_{n \geq 0}$. Cheon and Hahn [5] considered valuations of the division polynomials. Stange [27] gave a complete description of formulas for explicit valuations of division polynomials at primes of good or bad reduction.

In [11], the author and Bizim considered periodicity properties and $p$-adic properties of the sequences $\left(G_{n}(P)\right)_{n \geq 0}$ and $\left(H_{n}(P)\right)_{n \geq 0}$ of values of the division polynomials of an elliptic curve $E$ defined over a field $K$ evaluated at a point $P$. The authors showed that the sequences $\left(G_{n}(P)\right)_{n \geq 0}$ and $\left(H_{n}(P)\right)_{n \geq 0}$ are periodic when $K$ is a finite field. Then 
they proved that certain subsequences of these sequences converge when $K$ is a local field similar to that of the sequence $\left(F_{n}(P)\right)_{n \geq 0}$. In [8, Theorem 3.2], the author gave closed formulas in terms of the coefficients of the Weierstrass normal forms for sequences $\left(F_{n}(P)\right)_{n \geq 0}$ associated to elliptic curves with cyclic torsion subgroups and determined square and cube terms in these sequences. Stange [27, Section 12.2], demonstrated a method to obtain similar formulas by using other methods. In [9], the author showed that the coefficients of an elliptic curve $E$ can be given in terms of the sequences $\left(G_{n}(P)\right)_{n \geq 0}$ and $\left(H_{n}(P)\right)_{n \geq 0}$, and gave similar formulas for sequences $\left(G_{n}(P)\right)_{n \geq 0}$ and $\left(H_{n}(P)\right)_{n \geq 0}$ associated to an elliptic curve $E$ with cyclic torsion subgroup.

Although the sequences associated to elliptic curves with cyclic torsion subgroup have been dealt with by authors, the non-cyclic case was not considered. The present paper is motivated by the desire to contribute to the completeness of the literature by studying the non-cyclic cases. Here, we consider the sequences $\left(F_{n}(P)\right)_{n \geq 0},\left(G_{n}(P)\right)_{n \geq 0}$, and $\left(H_{n}(P)\right)_{n \geq 0}$ of values of the division polynomials of an elliptic curve with non-cyclic torsion subgroup and give explicit formulas for these sequences. As applications, we obtain formulas for elliptic divisibility sequences associated to elliptic curves with non-cyclic torsion subgroup and we determine square terms in these sequences.

\section{The sequences $\left(F_{n}(P)\right)_{n \geq 0},\left(G_{n}(P)\right)_{n \geq 0}$, and $\left(H_{n}(P)\right)_{n \geq 0}$ associated to elliptic curves with non-cyclic torsion subgroup}

The Mordell-Weil theorem states that if $K$ is a number field, then the group $E(K)$ is finitely generated. In particular, it is very important to characterize the torsion subgroups of $E(K)$. A complete list of possible torsion subgroups for rational elliptic curves is given by the following theorem due to Mazur.

Theorem $2.1([15])$. Let $E$ be an elliptic curve defined over $\mathbb{Q}$. Then the torsion subgroup $E_{\text {tors }}(\mathbb{Q})$ of $E$ is either isomorphic to $\mathbb{Z} / N \mathbb{Z}$ for $N=1,2, \cdots, 10,12$ or to $\mathbb{Z} / 2 \mathbb{Z} \times \mathbb{Z} / 2 N \mathbb{Z}$ for $N=1,2,3,4$. Further, each of these groups does occur as an $E_{\text {tors }}(\mathbb{Q})$.

The elliptic curves with the specified torsion subgroup lie in a one parameter family, see [13] for explicit parameterizations. In [8, Theorem 3.2], the author considers elliptic curves having cyclic torsion subgroups and gives explicit formulas for the sequences $\left(F_{n}(P)\right)_{n \geq 0}$ associated to these curves. Then in [9, Section 4, and Appendix A], the author obtains similar formulas for the sequences $\left(G_{n}(P)\right)_{n \geq 0}$ and $\left(H_{n}(P)\right)_{n \geq 0}$ associated to elliptic curves having cyclic torsion subgroups. In this paper we consider sequences associated to elliptic curves having non-cyclic torsion subgroup. We will obtain many results for these sequences similar to those for sequences associated to elliptic curves with cyclic torsion subgroups.

The following theorem gives parameterizations of elliptic curves with non-cyclic torsion subgroups, see [13] and see also [12].

Theorem 2.2. Let $E$ be an elliptic curve defined over $\mathbb{Q}$, and let the torsion subgroup $E_{\text {tors }}(\mathbb{Q})$ of $E$ be isomorphic to $\mathbb{Z} / 2 \mathbb{Z} \times \mathbb{Z} / 2 N \mathbb{Z}$. Then $E$ can be given in the following normal form

$$
E_{N}: y^{2}+(1-c) x y-b y=x^{3}-b x^{2}
$$

with following relations:

1. If $N=2$, then $b=\left(v^{2}-1\right) / 16, v \neq 0, \pm 1 / 4, c=0$.

2. If $N=3$, then $b=c+c^{2}, c=(10-2 \alpha) /\left(\alpha^{2}-9\right), c^{6}(c+1)^{3}(9 c+1) \neq 0$.

3. If $N=4$, then $b=(2 d-1)(d-1), c=(2 d-1)(d-1) / d, d=\alpha(8 \alpha+2) /\left(8 \alpha^{2}-1\right)$, $d(d-1)(2 d-1)\left(8 d^{2}-8 d+1\right) \neq 0$.

In particular if $N=1$, then

$$
E_{1}: y^{2}=x(x+k)(x+l)
$$


$k, l \neq 0$ and $k \neq l$.

Theorem 2.2 tells that every elliptic curve having a non-cyclic torsion subgroup isomorphic to $\mathbb{Z} / 2 \mathbb{Z} \times \mathbb{Z} / 2 N \mathbb{Z}$ for $N=1,2,3,4$ is birationally equivalent to one of the normal forms given above. We also note that in all cases the point $P=(0,0)$ is a torsion point of maximal order.

In this work we will assume that the coefficients of $E_{N}$ are integers for $N=1,2,3,4$. Then $k, l \in \mathbb{Z}$ for the case $N=1$, and we transform $E_{N}$ into a birationally equivalent curve $E_{N}^{\prime}$ having an equation with integral coefficients, for the cases $N=2,3,4$. The equations of the birationally equivalent curves for $N=2,3,4$ are given, respectively, as follows:

$$
\begin{aligned}
& E_{2}^{\prime}: y^{2}+16 x y-4^{4}\left(16 v^{2}-1\right) y=x^{3}-16\left(16 v^{2}-1\right) x^{2}, \\
& E_{3}^{\prime}: y^{2}+\left(\alpha^{2}+2 \alpha-19\right) \beta x y+\beta^{4} \gamma=x^{3}+\beta^{2} \gamma, \\
& E_{4}^{\prime}: y^{2}+\left(64 \alpha^{4}-24 \alpha^{2}-8 \alpha-1\right) \delta x y-(2 \alpha+1) \zeta^{3} \theta^{4} y=x^{3}-(2 \alpha+1) \zeta^{2} \theta^{2},
\end{aligned}
$$

where

$$
\beta=\alpha^{2}-9, \gamma=2 \alpha^{3}-14 \alpha^{2}+22 \alpha-10
$$

and

$$
\delta=8 \alpha^{2}-1, \zeta=\alpha(8 \alpha+2), \theta=8 \alpha^{2}+4 \alpha+1 .
$$

From now on, for simplicity of notation, we write $E_{2}, E_{3}, E_{4}$ for $E_{2}^{\prime}, E_{3}^{\prime}, E_{4}^{\prime}$, respectively.

We first consider the sequence $\left(F_{n}(P)\right)_{n \geq 0}$ of values of the division polynomials of an elliptic curve with non-cyclic torsion subgroup. In the following theorem, we give closed formulas in terms of the coefficients of the normal form $E_{N}$ associated to the sequence $\left(F_{n}(P)\right)_{n \geq 0}$, i.e., general term of the sequence $\left(F_{n}(P)\right)_{n \geq 0}$.

Theorem 2.3. Let $E_{N}$ be a normal form of an elliptic curve with non-cyclic torsion subgroup and let $\left(F_{n}(P)\right)_{n \geq 0}$ be the sequence of values of the $n$-division polynomials of $E_{N}$ at $P=(0,0)$. Let $\beta, \zeta, \delta$, and $\theta$ be as in (2.1) and (2.2). Then the general term of the sequence $\left(F_{n}(P)\right)_{n \geq 0}$ can be given by the following formulas:

1. If $N=1$, then

$$
F_{n}=\left\{\begin{array}{cl}
0, & \text { if } n \text { is even } \\
\varepsilon(k l)^{\left\{\left(n^{2}-1\right) / 4\right\},}, & \text { if } n \text { is odd, }
\end{array}\right.
$$

where

$$
\varepsilon= \begin{cases}+1, & \text { if } n \equiv 1(4) \\ -1, & \text { if } n \equiv 3(4)\end{cases}
$$

2. If $N=2$, then

$$
F_{n}=\left\{\begin{array}{cc}
0, & \text { if } n \equiv 0(4) \\
\varepsilon 2^{\left\{\left(5 n^{2}-p\right) / 2\right\}}\left(16 v^{2}-1\right)^{\left\{\left(3 n^{2}-q\right) / 8\right\}}, & \text { otherwise, }
\end{array}\right.
$$

where

$$
\varepsilon= \begin{cases}+1, & \text { if } n \equiv 1,5,6(8) \\ -1, & \text { if } n \equiv 2,3,7(8)\end{cases}
$$

and

$$
p=\left\{\begin{array}{ll}
5, & \text { if } n \equiv 1,3(4) \\
4, & \text { if } n \equiv 2(4)
\end{array} q= \begin{cases}3, & \text { if } n \equiv 1,3(4) \\
4, & \text { if } n \equiv 2(4)\end{cases}\right.
$$

3. If $N=3$, then

$$
F_{n}=\left\{\begin{array}{cc}
0, & \text { if } n \equiv 0(6) \\
\varepsilon(2 \alpha-10)^{\left\{\left(5 n^{2}-p\right) / 12\right\}}(\alpha-1)^{\left\{\left(2 n^{2}-q\right) / 3\right\}} \beta^{\left\{\left(5 n^{2}-r\right) / 4\right\},} & \text { otherwise, }
\end{array}\right.
$$


where

$$
\varepsilon= \begin{cases}+1, & \text { if } n \equiv 1,2,3,4,5(12) \\ -1, & \text { if } n \equiv 7,8,9,10,11(12),\end{cases}
$$

and

$$
p=\left\{\begin{array}{ll}
5, & \text { if } n \equiv 1,5(6) \\
8, & \text { if } n \equiv 2,4(6) \\
9, & \text { if } n \equiv 3(6),
\end{array} \quad q=\left\{\begin{array}{ll}
0, & \text { if } n \equiv 3(6) \\
2, & \text { otherwise, }
\end{array} \quad r= \begin{cases}5, & \text { if } n \equiv 1,3,5(6) \\
4, & \text { if } n \equiv 2,4(6) .\end{cases}\right.\right.
$$

4. If $N=4$, then

$$
F_{n}=\left\{\begin{array}{cc}
0, & \text { if } n \equiv 0(8) \\
\varepsilon(2 \alpha+1)^{\left\{\left(7 n^{2}-p\right) / 16\right\}} \zeta^{\left\{\left(15 n^{2}-q\right) / 16\right\}} \delta^{\left\{\left(5 n^{2}-r\right) / 4\right\}} \theta^{\left\{\left(3 n^{2}-s\right) / 8\right\},}, & \text { otherwise, }
\end{array}\right.
$$

where

$$
\varepsilon= \begin{cases}+1, & \text { if } n \equiv 1,4,5,9,10,13,14(16) \\ -1, & \text { if } n \equiv 2,3,6,7,11,12,15(16)\end{cases}
$$

and

$$
\begin{gathered}
p=\left\{\begin{array}{ll}
7, & \text { if } n \equiv 1,7(8) \\
12, & \text { if } n \equiv 2,6(8) \\
15, & \text { if } n \equiv 3,5(8) \\
16, & \text { if } n \equiv 4(8),
\end{array} \quad q= \begin{cases}15, & \text { if } n \equiv 1,7(8) \\
12, & \text { if } n \equiv 2,6(8) \\
7, & \text { if } n \equiv 3,5(8) \\
16, & \text { if } n \equiv 4(8),\end{cases} \right. \\
r=\left\{\begin{array}{ll}
5, & \text { if } n \equiv 1,3,5,7(8) \\
4, & \text { if } n \equiv 2,4,6(8),
\end{array} \quad s= \begin{cases}3, & \text { if } n \equiv 1,3,5,7(8) \\
4, & \text { if } n \equiv 2,6(8) \\
0, & \text { if } n \equiv 4(8) .\end{cases} \right.
\end{gathered}
$$

Proof. Before starting the proof, we note that if we take $n=2$, and then $m=n$ in equation (1.7) we have

$$
F_{n+2} F_{n-2}=F_{n+1} F_{n-1} F_{2}^{2}-F_{3} F_{n}^{2}
$$

since $F_{1}=1$.

We give the proof only for the case $N=3$, the other cases can be proved similarly. We argue by induction on $n$. First suppose that $n+1 \equiv 1$ (6) and the equation (2.5) is true for $n+1$. Then $n=6 k$ for some integer $k$ and we have to prove that

$$
F_{n+2}= \begin{cases}(2 \alpha-10)^{15 k^{2}+10 k+1}(\alpha-1)^{24 k^{2}+16 k+2} \beta^{45 k^{2}+30 k+4}, & \text { if } k \text { is even } \\ -(2 \alpha-10)^{15 k^{2}+10 k+1}(\alpha-1)^{24 k^{2}+16 k+2} \beta^{45 k^{2}+30 k+4}, & \text { if } k \text { is odd }\end{cases}
$$

On the other hand by assumption we have

$$
F_{2}=(2 \alpha-10)(\alpha-1)^{2} \beta^{4}, F_{3}=(2 \alpha-10)^{3}(\alpha-1)^{6} \beta^{10},
$$

and

and

$$
\begin{gathered}
F_{n}=0, \\
F_{n+1}= \begin{cases}(2 \alpha-10)^{15 k^{2}+5 k}(\alpha-1)^{24 k^{2}+8 k} \beta^{45 k^{2}+15 k}, & \text { if } k \text { is even } \\
-(2 \alpha-10)^{15 k^{2}+5 k}(\alpha-1)^{24 k^{2}+8 k} \beta^{45 k^{2}+15 k}, & \text { if } k \text { is odd },\end{cases} \\
F_{n-1}= \begin{cases}-(2 \alpha-10)^{15 k^{2}-5 k}(\alpha-1)^{24 k^{2}-8 k} \beta^{45 k^{2}-15 k}, & \text { if } k \text { is even } \\
(2 \alpha-10)^{15 k^{2}-5 k}(\alpha-1)^{24 k^{2}-8 k} \beta^{45 k^{2}-15 k}, & \text { if } k \text { is odd, }\end{cases}
\end{gathered}
$$

$$
F_{n-2}= \begin{cases}-(2 \alpha-10)^{15 k^{2}-10 k+1}(\alpha-1)^{24 k^{2}-16 k+2} \beta^{45 k^{2}-30 k+4}, & \text { if } k \text { is even } \\ (2 \alpha-10)^{15 k^{2}-10 k+1}(\alpha-1)^{24 k^{2}-16 k+2} \beta^{45 k^{2}-30 k+4}, & \text { if } k \text { is odd. }\end{cases}
$$

Substituting these expressions into (2.7), we obtain the equation (2.8). Thus we have proved the theorem is true for $n+2$ which completes the proof for $n \equiv 0$ (6). The other cases of the theorem can be proved by induction in the same way. 
We can use Thorem 2.3 to deduce general terms of elliptic divisibility sequences associated to elliptic curves with non-cyclic torsion group. An elliptic divisibility sequence (or EDS) $\left(h_{n}\right)_{n \geq 0}$ is a divisibility sequence satisfying a non-linear recurrence relation of the form

$$
h_{m+n} h_{m-n}=h_{m+1} h_{m-1} h_{n}^{2}-h_{n+1} h_{n-1} h_{m}^{2}
$$

for all $m \geq n \geq 1$. An elliptic divisibility sequence is called proper if $h_{0}=0, h_{1}=1$, $h_{2} h_{3} \neq 0$, and the discriminant of an $\operatorname{EDS}\left(h_{n}\right)_{n \geq 0}$ is the quantity

$$
\begin{aligned}
\Delta\left(h_{n}\right)= & h_{4} h_{2}^{15}-h_{3}^{3} h_{2}^{12}+3 h_{4}^{2} h_{2}^{10}-20 h_{4} h_{3}^{3} h_{2}^{7}+3 h_{4}^{3} h_{2}^{5} \\
& +16 h_{3}^{6} h_{2}^{4}+8 h_{4}^{2} h_{3}^{3} h_{2}^{2}+h_{4}^{4},
\end{aligned}
$$

(see [23] or [25], see also [30]). A proper EDS is said to be non-singular if $\Delta\left(h_{n}\right) \neq 0$. The arithmetic properties of EDSs were first studied by Ward in 1948 [30,31]. See also $[6,22,29]$ for more details on EDSs.

Ward defined the division polynomials over the field $\mathbb{C}$ and showed that non-singular elliptic divisibility sequences can be given in terms of elliptic functions by using the complex analytic theory of elliptic functions. More precisely, Ward [30, Theorem 12.1] proved that if $\left(h_{n}\right)_{n \geq 0}$ is a non-singular elliptic divisibility sequence, then there exist a lattice $L \subset \mathbb{C}$ and a complex number $z \in \mathbb{C}$ such that

$$
h_{n}=F_{n}(z, L)=\frac{\sigma(n z, L)}{\sigma(z, L)^{n^{2}}} \text { for all } n \geq 1,
$$

where $F_{n}(z, L)$ and $\sigma(z, L)$ are the $n$-division polynomial and the Weierstrass $\sigma$-function associated to the lattice $L$, respectively. Moreover Ward proved that the modular invariants $g_{2}(L)$ and $g_{3}(L)$ associated to the lattice $L$ and the Weierstrass values $\wp(z)$ and $\wp^{\prime}(z)$ associated to the point $z$ on the elliptic curve $\mathbb{C} / L$ can be given by the terms $h_{2}, h_{3}$, and $h_{4}$ of the sequence $\left(h_{n}\right)$, see [30, equations 13.6, 13.7, 13.5, and 13.1].

Silverman [23, Proposition 18] reformulated Ward's result to the case of rational numbers and proved that if $\left(h_{n}\right)_{n \geq 0}$ is a non-singular EDS, then there exists an elliptic curve $E$ given by a minimal Weierstrass equation over $\mathbb{Q}$ and a point $P \in E(\mathbb{Q})$ such that

$$
h_{n}=F_{n}(P) \quad \text { for all } n \geq 1 .
$$

Therefore EDSs can be defined via recurrence relation (2.9) or as division polynomials. It follows that Theorem 2.3 applies to the non-singular elliptic divisibility sequences $\left(h_{n}\right)_{n \geq 0}$ associated to elliptic curves with non-cyclic torsion group. Thus we can obtain general term formulas for non-singular elliptic divisibility sequences associated to elliptic curves with non-cyclic torsion group similar to formulas in Theorem 2.3.

Now we consider the sequence $\left(G_{n}(P)\right)_{n \geq 0}$ of values of the division polynomials of an elliptic curve with non-cyclic torsion subgroup. In the following theorem we give general terms of the sequences $\left(G_{n}(P)\right)_{n \geq 0}$ associated to $E_{1}, E_{2}, E_{3}$, and $E_{4}$ respectively. The proof uses Theorem 2.3.

Theorem 2.4. Let $E_{N}$ be a normal form of an elliptic curve with non-cyclic torsion subgroup and let $\left(G_{n}(P)\right)_{n \geq 0}$ be the sequence of values of the $n$-division polynomials of $E_{N}$ at $P=(0,0)$. Let $\beta, \zeta, \delta$, and $\theta$ be as in (2.1) and (2.2). Then the general term of the sequence $\left(G_{n}(P)\right)_{n \geq 0}$ can be given by the following formulas:

1. If $N=1$, then

$$
G_{n}=\left\{\begin{array}{cc}
0, & \text { if } n \text { is odd } \\
(k l)^{\left\{n^{2} / 2\right\}}, & \text { if } n \text { is even. }
\end{array}\right.
$$

2. If $N=2$, then

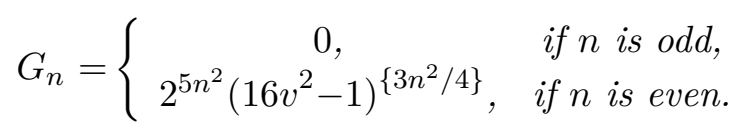


3. If $N=3$, then

$$
G_{n}=\left\{\begin{array}{cc}
0, & \text { if } n \equiv 1,5(6) \\
\varepsilon(2 \alpha-10)^{\left\{\left(5 n^{2}-p\right) / 6\right\}}(\alpha-1)^{\left\{\left(4 n^{2}+q\right) / 3\right\}} \beta^{\left\{\left(5 n^{2}+r\right) / 2\right\}}, & \text { otherwise, }
\end{array}\right.
$$

where

$$
\varepsilon= \begin{cases}+1, & \text { if } n \equiv 0(6) \\ -1, & \text { otherwise }\end{cases}
$$

and

$$
p=\left\{\begin{array}{ll}
0, & \text { if } n \equiv 0(6) \\
2, & \text { if } n \equiv 2,4(6) \\
3, & \text { if } n \equiv 3(6),
\end{array} \quad q=\left\{\begin{array}{ll}
0, & \text { if } n \equiv 0,3(6) \\
2, & \text { otherwise, }
\end{array} \quad r= \begin{cases}0, & \text { if } n \equiv 0,2,4(6) \\
1, & \text { if } n \equiv 3(6) .\end{cases}\right.\right.
$$

4. If $N=4$, then

$$
G_{n}=\left\{\begin{array}{cc}
0, & \text { if } n \equiv 1,7(8) \\
(2 \alpha+1)^{\left\{\left(7 n^{2}-p\right) / 8\right\}} \zeta^{\left\{\left(15 n^{2}+q\right) / 8\right\}} \delta^{\left\{\left(5 n^{2}+r\right) / 2\right\}} \theta^{\left\{\left(3 n^{2}+r\right) / 4\right\},}, & \text { otherwise, }
\end{array}\right.
$$

where

$$
p=\left\{\begin{array}{ll}
0, & \text { if } n \equiv 0(8) \\
4, & \text { if } n \equiv 2,6(8) \\
7, & \text { if } n \equiv 3,5(8) \\
8, & \text { if } n \equiv 4(8),
\end{array} \quad q= \begin{cases}0, & \text { if } n \equiv 0(8) \\
4, & \text { if } n \equiv 2,6(8) \\
1, & \text { if } n \equiv 3,5(8) \\
8, & \text { if } n \equiv 4(8)\end{cases}\right.
$$

and

$$
r= \begin{cases}0, & \text { if } n \equiv 0,2,4,6(8) \\ 1, & \text { if } n \equiv 3,5(8)\end{cases}
$$

Proof. We give the proof only for the case $N=3$, the other cases can be proved similarly.

Let $n \equiv 1$ (6) and write $n=6 k+1, k \geq 0$. Then by (1.5)

$$
G_{6 k+1}=-F_{6 k+2} F_{6 k} \text { for all } k \geq 0,
$$

since $x=0$. Hence $G_{6 k+1}=0$, since $F_{6 k}=0$ for all $k \geq 0$.

Now let $n \equiv 2(6)$. Then by (1.5)

$$
G_{6 k+2}=-F_{6 k+1} F_{6 k+3} \text { for all } k \geq 0,
$$

since $x=0$. On the other hand we have

$$
F_{6 k+1}= \begin{cases}(2 \alpha-10)^{15 k^{2}+5 k}(\alpha-1)^{24 k^{2}+8 k} \beta^{45 k^{2}+15 k}, & \text { if } k \text { is even } \\ -(2 \alpha-10)^{15 k^{2}+5 k}(\alpha-1)^{24 k^{2}+8 k} \beta^{45 k^{2}+15 k}, & \text { if } k \text { is odd }\end{cases}
$$

and

$$
F_{6 k+3}= \begin{cases}(2 \alpha-10)^{15 k^{2}+5 k+3}(\alpha-1)^{24 k^{2}+24 k+6} \beta^{45 k^{2}+45 k+10}, & \text { if } k \text { is even } \\ -(2 \alpha-10)^{15 k^{2}+5 k+3}(\alpha-1)^{24 k^{2}+24 k+6} \beta^{45 k^{2}+45 k+10}, & \text { if } k \text { is odd, }\end{cases}
$$

by (2.5). Now substituting these expressions into (2.16) we derive that

$$
G_{6 k+2}=-(2 \alpha-10)^{30 k^{2}+20 k+3}(\alpha-1)^{48 k^{2}+32 k+6} \beta^{90 k^{2}+60 k+10}
$$

for all $k \geq 0$. On the other hand by the formula (2.14) we have

$$
G_{6 k+2}=-(2 \alpha-10)^{30 k^{2}+20 k+3}(\alpha-1)^{48 k^{2}+32 k+6} \beta^{90 k^{2}+60 k+10},
$$

which completes the proof for $n \equiv 2(6)$. The remaining cases can be proved in a similar manner. 
Finally we consider the sequence $\left(H_{n}(P)\right)_{n>0}$ of values of the division polynomials of an elliptic curve with non-cyclic torsion subgroup. In the following theorem we give the general terms of the sequence $\left(H_{n}(P)\right)_{n>0}$ associated to elliptic curves $E_{2}, E_{3}$, and $E_{4}$ respectively. We note that the sequence $\left(H_{n}(P)\right)_{n>0}$ associated to elliptic curve $E_{1}$ is not defined since $F_{2}=0$; see relation (1.6). The proof of the theorem is similar to the proof of Theorem 2.4.

Theorem 2.5. Let $E_{N}$ be a normal form of an elliptic curve with non-cyclic torsion subgroup and let $\left(H_{n}(P)\right)_{n \geq 0}$ be the sequence of values of the $n$-division polynomials of $E_{N}$ at $P=(0,0)$. Let $\beta, \zeta, \delta$, and $\theta$ be as in (2.1) and (2.2). Then the general term of the sequence $\left(H_{n}(P)\right)_{n \geq 0}$ can be given by the following formulas:

1. If $N=2$, then

$$
H_{n}=\left\{\begin{array}{cc}
0, & \text { if } n \equiv 1,2(4) \\
\varepsilon 2^{\left\{\left(15 n^{2}+p\right) / 2\right\}}\left(16 v^{2}-1\right)^{\left\{\left(9 n^{2}-p\right) / 8\right\}}, & \text { otherwise }
\end{array}\right.
$$

where

$$
\varepsilon= \begin{cases}+1, & \text { if } n \equiv 0(8) \\ -1, & \text { if } n \equiv 3,4,7(8),\end{cases}
$$

and

$$
p= \begin{cases}0, & \text { if } n \equiv 0(4) \\ 1, & \text { if } n \equiv 3(4) .\end{cases}
$$

2. If $N=3$, then

$$
H_{n}=\left\{\begin{array}{cc}
0, & \text { if } n \equiv 1,4(6) \\
\varepsilon(2 \alpha-10)^{\left\{\left(5 n^{2}-p\right) / 4\right\}}(\alpha-1)^{2 n^{2}} \beta^{\left\{\left(15 n^{2}+p\right) / 4\right\},} & \text { otherwise, }
\end{array}\right.
$$

where

$$
\varepsilon= \begin{cases}+1, & \text { if } n \equiv 0,2,3,11(12) \\ -1, & \text { if } n \equiv 5,6,8,9(12),\end{cases}
$$

and

$$
p= \begin{cases}0, & \text { if } n \equiv 0,2(6) \\ 1, & \text { if } n \equiv 3,5(6)\end{cases}
$$

3. If $N=4$, then

$$
H_{n}=\left\{\begin{array}{cc}
0, & \text { if } n \equiv 1,6(8) \\
\varepsilon(2 \alpha+1)^{\left\{\left(21 n^{2}-p\right) / 16\right\}} \zeta^{\left\{\left(45 n^{2}+q\right) / 16\right\}} \delta^{\left\{\left(15 n^{2}+r\right) / 4\right\}} \theta^{\left\{\left(9 n^{2}+s\right) / 8\right\}}, & \text { otherwise, }
\end{array}\right.
$$

where

$$
\varepsilon= \begin{cases}+1, & \text { if } n \equiv 0,4,5,10,13(16) \\ -1, & \text { if } n \equiv 2,3,7,8,11,12,15(16),\end{cases}
$$

and

$$
\begin{gathered}
p=\left\{\begin{array}{cc}
0, & \text { if } n \equiv 0(8) \\
4, & \text { if } n \equiv 2(8) \\
13, & \text { if } n \equiv 3,5(8) \\
16, & \text { if } n \equiv 4(8) \\
5, & \text { if } n \equiv 7(8),
\end{array} \quad q=\left\{\begin{array}{cc}
0, & \text { if } n \equiv 0(8) \\
-4, & \text { if } n \equiv 2(8) \\
11, & \text { if } n \equiv 3(8) \\
16, & \text { if } n \equiv 4(8) \\
-5, & \text { if } n \equiv 5(8) \\
3, & \text { if } n \equiv 7(8),
\end{array}\right.\right. \\
r=\left\{\begin{array}{cc}
0, & \text { if } n \equiv 0,2,4(8) \\
1, & \text { if } n \equiv 3,5,7(8),
\end{array} \quad s=\left\{\begin{array}{cc}
0, & \text { if } n \equiv 0,4(8) \\
4, & \text { if } n \equiv 2(8) \\
-1, & \text { if } n \equiv 3,7(8) \\
7, & \text { if } n \equiv 5(8) .
\end{array}\right.\right.
\end{gathered}
$$




\section{Squares in sequences associated to elliptic curves with non-cyclic tor- sion subgroups}

The problem of determining square terms in linear recurrence sequences is of great interest in the literature [3,4,19-21]. The authors have also considered the square terms in elliptic divisibility sequences $[8,10]$, see also $[16,18]$. In $[8$, Section 5$]$, the author determined square and cube terms in the sequence $\left(F_{n}(P)\right)_{n \geq 0}$ associated to a normal form of an elliptic curve with cyclic torsion subgroup. In [9, Section 5, and Appendix B], the author considered similar problems for the sequences $\left(G_{n}(P)\right)_{n \geq 0}$, and $\left(H_{n}(P)\right)_{n \geq 0}$ associated to an elliptic curve with cyclic torsion subgroup. In this paper we determine square terms in sequences associated to elliptic curves having non-cyclic torsion subgroup.

Throughout this paper, $\square= \pm \eta^{2}$ where $\eta$ is a non-zero integer. In the following theorems we will answer the following three questions:

(1) Which terms of the sequence $\left(F_{n}(P)\right)_{n \geq 0}$ (resp. $\left.\left(G_{n}(P)\right)_{n \geq 0},\left(H_{n}(P)\right)_{n \geq 0}\right)$ must be $\square$ independent of parameters $k, l, v$, and $\alpha$ determining the coefficients of the curve $E_{N}$ ?

(2) Which terms of the sequence $\left(F_{n}(P)\right)_{n \geq 0}$ (resp. $\left.\left(G_{n}(P)\right)_{n \geq 0},\left(H_{n}(P)\right)_{n \geq 0}\right)$ can be $\square$ with admissible choice of parameters $k, l, v$, and $\alpha$ ?

(3) Which terms of the sequence $\left(F_{n}(P)\right)_{n \geq 0}$ (resp. $\left.\left(G_{n}(P)\right)_{n \geq 0},\left(H_{n}(P)\right)_{n \geq 0}\right)$ cannot be $\square$ independent of parameters $k, l, v$, and $\alpha$ ?

We first answer these questions for the sequence $\left(F_{n}(P)\right)_{n \geq 0}$, in the following theorem. The proof of the theorem uses solutions of some Diophantine equations.

Theorem 3.1. Let $E_{N}$ be a normal form of an elliptic curve with non-cyclic torsion subgroup, let $\left(F_{n}(P)\right)_{n \geq 0}$ be the sequence of values of the $n$-division polynomials of $E_{N}$ at $P=(0,0)$, and let $F_{n}(\bar{P}) \neq 0$.

1. Let $N=1$. Then $F_{n}=\square$ for all odd $n$, and all non-zero $k, l$.

2. Let $N=2$.

- If $n \equiv 1,7$ (8), then $F_{n}=\square$ for all $v$,

- otherwise $F_{n} \neq \square$ for all $v$.

3. Let $N=3$.

- If $n \equiv 1,5,7,11$ (12), then $F_{n}=\square$ for all $\alpha \neq-3,1,3,5$,

- if $n \equiv 2,3,9,10(12)$, then $F_{n}=\square$ if and only if $2(\alpha-5)=\square$,

- otherwise $F_{n} \neq \square$ for all $\alpha$.

4. Let $N=4$.

- If $n \equiv 1,15$ (16), then $F_{n}=\square$ for all non-zero $\alpha$,

- otherwise $F_{n} \neq \square$ for all $\alpha$.

Proof. We shall prove the theorem only for the case $N=4$ as the proofs of the other cases can easily be obtained. We note that $F_{n}=0$ for $n \equiv 0,8(16)$, by (2.6). It can easily be seen that if $n \equiv 1,15$ (16), then $F_{n}=\square$ for all non-zero $\alpha$, by using (2.6).

If $n \equiv 2,6,10,14(16)$, then $F_{n}=\square$ if and only if

$$
2 \alpha(2 \alpha+1)(4 \alpha+1)\left(8 \alpha^{2}+4 \alpha+1\right)=\square
$$

by (2.6). It can easily be seen that the irreducible factors in equation (3.1) are pairwise relatively prime*. Thus the last equation leads to

$$
2 \alpha(2 \alpha+1)=\square
$$

\footnotetext{
*Note that $2 \alpha$ cannot have a common prime factor $p$ with any of $2 \alpha+1,4 \alpha+1,8 \alpha^{2}+4 \alpha+1$, since $2 \alpha \equiv 0(p)$ implies that $2 \alpha+1,4 \alpha+1$ and $8 \alpha^{2}+4 \alpha+1$ are $\equiv 1(p)$. Next, $2 \alpha+1$ cannot have a common prime factor $p$ with neither $4 \alpha+1$ nor $8 \alpha^{2}+4 \alpha+1$, since $p$ should be odd, so $\alpha \equiv-1 / 2(p)$ and, consequently $4 \alpha+1 \equiv-1(p)$ and $8 \alpha^{2}+4 \alpha+1 \equiv 1(p)$. Finally $4 \alpha+1$ and $8 \alpha^{2}+4 \alpha+1$ cannot have a common prime factor $p$, because $p$ should be odd, thus $\alpha \equiv-1 / 4 \quad(p)$ and so $8 \alpha^{2}+4 \alpha+1$ $\equiv 1 / 2(p)$.
} 
and using the fact that "if $x y=\square$, then $(x+y)^{2}-(x-y)^{2}=\square$ " to obtain trivial equations

$$
(4 \alpha+1)^{2} \pm \eta^{2}=1 \text {, }
$$

where $\eta$ is a non-zero integer. It is clear that the solutions of these equations do not provide any acceptable $\alpha$.

If $n \equiv 3,13$ (16), then $F_{n}=\square$ if and only if

$$
(2 \alpha+1)\left(8 \alpha^{2}+4 \alpha+1\right)=
$$

by (2.6). This equation leads to equations

$$
(4 \alpha)^{3}+4(4 \alpha)^{2}+6(4 \alpha)+4=\eta^{2}
$$

or

$$
(-4 \alpha)^{3}-4(-4 \alpha)^{2}+6(-4 \alpha)-4=\eta^{2}
$$

where $\eta$ is a non-zero integer. The first equation gives an elliptic curve with rank 1. Applying the Elliptic Logarithm Method ${ }^{\dagger}$ we see that the integer solutions of this equation are $(4 \alpha, \eta)=(-2,0),(-1, \pm 1),(0, \pm 2),(6, \pm 20)$, and these points do not provide any acceptable $\alpha$. The second equation gives an elliptic curve with rank zero ${ }^{\ddagger}$ and the integer point on this curve does not provide any acceptable $\alpha$.

If $n \equiv 4,12(16)$, then $F_{n}=\square$ if and only if

$$
8 \alpha^{2}-1=
$$

by (2.6), and this equation leads to

$$
(4 \alpha)^{2}-2 \eta^{2}=2
$$

or

$$
(4 \alpha)^{2}+2 \eta^{2}=2
$$

The first equation is a Pell equation and the solutions of this equation are $(4 \alpha, \eta)=(2,1)$, $(10,7),(58,41), \ldots$ and these solutions do not give desired $\alpha$ since $4 \alpha \equiv 2$ (4). The solutions of the latter equation do not provide any acceptable $\alpha$.

If $n \equiv 5,11$ (16), then $F_{n}=\square$ if and only if

$$
2 \alpha(4 \alpha+1)\left(8 \alpha^{2}+4 \alpha+1\right)=
$$

by (2.6), or equivalently

$$
2 \alpha\left(8 \alpha^{2}+4 \alpha+1\right)=\square .
$$

This equation leads to equations

$$
(4 \alpha)^{3}+2(4 \alpha)^{2}+2(4 \alpha)=\eta^{2}
$$

or

$$
(-4 \alpha)^{3}-2(-4 \alpha)^{2}+2(-4 \alpha)=\eta^{2}
$$

where $\eta$ is a non-zero integer. These equations give elliptic curves with rank 1 and applying the Elliptic Logarithm Method we see that the integer points on these curves do not provide any acceptable $\alpha$.

Finally if $n \equiv 7,9$ (16), then $F_{n}=\square$ if and only if

$$
2 \alpha(2 \alpha+1)(4 \alpha+1)=\square .
$$

This equation leads to equations

$$
(4 \alpha)^{3}+3(4 \alpha)^{2}+2(4 \alpha)=\eta^{2}
$$

${ }^{\dagger}$ This has been developed in [28] and, independently, in [7] and now is implemented in MAGMA [14]; see also [2].

¥The only solutions are those given by coordinates of the torsion points. These, in turn, can be computed by the Lutz-Nagell Theorem (see, for example, Corollary 7.2, Chapter VIII.7 of [24]); automatically, they can be calculated using e.g. the PARI-GP calculator [17] or the online MAGMA calculator [14]. 
or

$$
(-4 \alpha)^{3}-3(-4 \alpha)^{2}+2(-4 \alpha)=\eta^{2},
$$

where $\eta$ is a non-zero integer. These equations give elliptic curves with rank 0 and the integer points on these curves do not provide any acceptable $\alpha$.

The following theorem gives the squares in the sequences $\left(G_{n}(P)\right)_{n \geq 0}$ associated to $E_{1}$, $E_{2}, E_{3}$, and $E_{4}$ respectively. The proof is similar to proof of Theorem 3.1.

Theorem 3.2. Let $E_{N}$ be a normal form of an elliptic curve with non-cyclic torsion group and let $\left(G_{n}(P)\right)_{n \geq 0}$ be the sequence of values of the $n$-division polynomials of $E_{N}$ at $P=(0,0)$, and let $G_{n}(P) \neq 0$.

1. Let $N=1$. Then $G_{n}=\square$ for all even $n$, and all non-zero $k, l$.

2. Let $N=2$.

- If $n \equiv 0$ (4), then $G_{n}=\square$ for all $v$,

- otherwise $G_{n} \neq \square$ for all $v$.

3. Let $N=3$.

- If $n \equiv 0$ (6), then $G_{n}=\square$ for all $\alpha \neq-3,1,3,5$,

- if $n \equiv 2,4(6)$, then $G_{n}=\square$ if and only if $2(\alpha-5)=\square$,

- otherwise $G_{n} \neq \square$ for all $\alpha$.

4. Let $N=4$.

- If $n \equiv 0$ (8), then $G_{n}=\square$ for all non-zero $\alpha$,

- otherwise $G_{n} \neq \square$ for all $\alpha$.

The following theorem gives the squares in the sequences $\left(H_{n}(P)\right)_{n \geq 0}$ associated to $E_{2}$, $E_{3}$ and $E_{4}$ respectively.

Theorem 3.3. Let $E_{N}$ be a normal form of an elliptic curve with non-cyclic torsion group and let $\left(H_{n}(P)\right)_{n \geq 0}$ be the sequence of values of the $n$-division polynomials of $E_{N}$ at $P=(0,0)$, and let $H_{n}(P) \neq 0$.

1. Let $N=2$.

- If $n \equiv 0,3,4$ (8), then $H_{n}=\square$ for all $v$,

- otherwise $H_{n} \neq \square$ for all $v$.

2. Let $N=3$.

- If $n \equiv 0,8$ (12), then $H_{n}=\square$ for all $\alpha \neq-3,1,3,5$,

- if $n \equiv 3,4,5,9,11(12)$, then $H_{n}=\square$ if and only if $2(\alpha-5)=\square$,

- otherwise $H_{n} \neq \square$ for all $\alpha$.

3. Let $N=4$.

- If $n \equiv 0,4,8,12$ (16), then $H_{n}=\square$ for all non-zero $\alpha$,

- if $n \equiv 3(16)$, then $H_{n}=\square$ if and only if $2 \alpha+1=\square$,

- if $n \equiv 5,7$ (16), then $H_{n}=\square$ if and only if $8 \alpha^{2}+4 \alpha+1=\square$,

- otherwise $H_{n} \neq \square$ for all $\alpha$.

Proof. We shall prove the theorem only for the case $N=4$ as the proofs of the other cases are entirely similar. We note that $H_{n}=0$ for $n \equiv 1,6,9$ (16) and if $n \equiv 0,4,8$, 12 (16), then $H_{n}=\square$ for all non-zero $\alpha$, by (2.19).

If $n \equiv 2,10(16)$, then $H_{n}=\square$ if and only if

$$
2 \alpha(2 \alpha+1)(4 \alpha+1)\left(8 \alpha^{2}-1\right)\left(8 \alpha^{2}+4 \alpha+1\right)=\square,
$$

if $n \equiv 11(16)$, then $H_{n}=\square$ if and only if

$$
2 \alpha(4 \alpha+1)=\square,
$$

and if $n \equiv 13,15$ (16), then $H_{n}=\square$ if and only if

$$
2 \alpha(2 \alpha+1)(4 \alpha+1)\left(8 \alpha^{2}+4 \alpha+1\right)=\square .
$$


We see that the factors appearing in the left hand side of these equations are relatively prime, and so every factor is $\square$. Thus the equations (3.4), (3.5), and (3.6) lead to

$$
(4 \alpha+1)^{2} \pm \eta^{2}=1 \text {, }
$$

where $\eta$ is a non-zero integer. It is clear that the solutions of these equations do not provide any acceptable $\alpha$.

If $n \equiv 3$ (16), then $H_{n}=\square$ if and only if $2 \alpha+1=\square$, by (2.19).

Finally if $n \equiv 5,7(16)$, then $H_{n}=\square$ if and only if $8 \alpha^{2}+4 \alpha+1=\square$. Hence $H_{n}=$ if and only if

$$
(4 \alpha+1)^{2}-2 \eta^{2}=-1
$$

or

$$
(4 \alpha+1)^{2}+2 \eta^{2}=-1
$$

where $\eta$ is a non-zero integer. The last equation is impossible. The first equation leads to Pell equation and the least solution of this equation is $(4 \alpha+1, \eta)=(1,1)$ leading to $(\alpha$, $\eta)=(0,1)$. Thus the equation has infinitely many solutions and half of these solutions are congruent to 1 modulo 4 , but not all. It is clear that the solutions that are congruent to 1 modulo 4 gives acceptable $\alpha$.

Acknowledgment. This work was supported by the research fund of Bursa Uludağ University project no: $\mathrm{KUAP}(\mathrm{F})-2017 / 3$.

\section{References}

[1] M. Ayad, Périodicité ( $\bmod q)$ des suites elliptiques et points $S$-entiers sur les courbes elliptiques, Ann. Inst. Fourier, 43 (3), 585-618, 1993.

[2] W. Bosma, J. Cannon, and C. Playoust, The Magma Algebra System I. The user language, J. Symbolic Comput. 24 (3-4), 235-265, 1997.

[3] A. Bremner and N. Tzanakis, Lucas sequences whose 12th or 9th term is a square, J. Number Theory, 107, 215-227, 2004.

[4] A. Bremner and N. Tzanakis, On squares in Lucas sequences , J. Number Theory, 124, 511-520, 2007.

[5] J. Cheon and S. Hahn, Explicit valuations of division polynomials of an elliptic curve, Manuscripta Math. 97, 319-328, 1998.

[6] G. Everest, A. van der Poorten, I. Shparlinski, and T. Ward, Recurrence Sequences, Math. Surveys Monogr. 104, AMS, Providence, RI, 2003.

[7] J. Gebel, A. Pethő, and H.G. Zimmer, Computing integral points on elliptic curves, Acta Arith. 68, 171-192, 1994.

[8] B. Gezer, Elliptic divisibility sequences, squares and cubes, Publ. Math. Debrecen, 83 (3), 481-515, 2013.

[9] B. Gezer, Sequences associated to elliptic curves, arXiv:1909.12654.

[10] B. Gezer and O. Bizim, Squares in elliptic divisibility sequences, Acta Arith. 144 (2), 125-134, 2010.

[11] B. Gezer and O. Bizim, Sequences generated by elliptic curves, Acta Arith. 188 (3), 253-268, 2019.

[12] D. Husemöller, Elliptic Curves, Springer Verlag, New York, 1987.

[13] D.S. Kubert, Universal bounds on the torsion of elliptic curves, Proc. London Math. Soc. 33 (3), 193-237, 1976.

[14] http://magma.maths.usyd.edu.au/calc/

[15] B. Mazur, Modular curves and the Eisenstein ideal, Publ. Math. IHES, 47, 33-186, 1977.

[16] V. Mahé, Prime power terms in elliptic divisibility sequences, Math. Comp. 83 (288), 1951-1991, 2014.

[17] http://pari.maths.u-bordeaux.fr/ 
[18] J. Reynolds, Perfect powers in elliptic divisibility sequences, J. Number Theory, 132 , 998-1015, 2012.

[19] P. Ribenboim, Pell numbers, squares and cubes, Publ. Math. Debrecen, 54, 131-152, 1999.

[20] P. Ribenboim and W. McDaniel, The square terms in Lucas sequences, J. Number Theory, 58, 104-123, 1996.

[21] P. Ribenboim and W. McDaniel, Squares in Lucas sequences having an even first parameter, Colloq. Math. 78, 29-34, 1998.

[22] R. Shipsey, Elliptic divisibility sequences, PhD thesis, Goldsmiths, University of London, 2000.

[23] J.H. Silverman, p-adic properties of division polynomials and elliptic divisibility sequences, Math. Ann. 332 (2), 443-471, 2005, addendum 473-474.

[24] J.H. Silverman, The Arithmetic of Elliptic Curves (2nd Edition), Graduate Texts in Mathematics, 106, Springer, Dordrecht, 2009.

[25] J.H. Silverman and N. Stephens, The sign of an elliptic divisibility sequence, J. Ramanujan Math. Soc. 21 (1), 1-17, 2006.

[26] J.H. Silverman and J. Tate, Rational Points on Elliptic Curves, Undergraduate Texts in Mathematics, Springer, 1992.

[27] K. Stange, Integral points on elliptic curves and explicit valuations of division polynomials, Canad. J. Math. 68 (5), 1120-1158, 2016.

[28] R.J. Stroeker, N. Tzanakis N, Solving elliptic diophantine equations by estimating linear forms in elliptic logarithms, Acta Arith. 67, 177-196, 1994.

[29] C.S. Swart, Elliptic curves and related sequences, PhD Thesis, Royal Holloway, University of London, 2003.

[30] M. Ward, Memoir on elliptic divisibility sequences, Amer. J. Math. 70, 31-74, 1948.

[31] M. Ward, The law of repetition of primes in an elliptic divisibility sequences, Duke Math. J. 15, 941-946, 1948. 